\title{
Identification of ter94, Drosophila VCP, as a modulator of polyglutamine-induced neurodegeneration
}

\author{
H Higashiyama ${ }^{1,2,5}$, F Hirose ${ }^{3}$, M Yamaguchi $^{3}$, YH Inoue ${ }^{3}$, \\ N Fujikake ${ }^{1,4}$, A Matsukage ${ }^{3}$ and A Kakizuka*,1,2 \\ 1 The Fourth Department, Osaka Bioscience Institute, 565-0874 Osaka, Japan \\ 2 CREST, JST (Japan Science and Technology Corporation) \\ ${ }^{3}$ Division of Biochemistry, Cell Biology Section, Aichi Cancer Center Research \\ Institute, 464-8681 Aichi, Japan \\ ${ }^{4}$ Graduate course, Kyoto University, Kyoto, Japan \\ ${ }^{5}$ Current address: Cold Spring Harbor Laboratory, New York, USA \\ * Corresponding author: A Kakizuka. Present address: Laboratory of Functional \\ Biology, Graduate School of Biostudies, Kyoto University, Kyoto 606-8501, \\ Japan. Tel: +81-75-753-7675; Fax: +81-75-753-7676; \\ E-mail: kakizuka@lif.kyoto-u.ac.jp
}

Received 30.7.01; accepted 5.9.01

Edited by $\mathrm{H}$ Ichijo

\begin{abstract}
We have successfully generated a Drosophila model of human polyglutamine (polyQ) diseases by the targeted expression of expanded-polyQ (ex-polyQ) in the Drosophila compound eye. The resulting eye degeneration is progressive and ex-polyQ dosage- and ex-poly $Q$ length-dependent. Furthermore, intergenerational changes in repeat length were observed in homozygotes, with concomitant changes in the levels of degeneration. Through genetic screening, using this fly model, we identified loss-of-function mutants of the ter94 gene that encodes the Drosophila homolog of VCP/CDC48, a member of the AAA+ class of the ATPase protein family, as dominant suppressors. The suppressive effects of the ter94 mutants on ex-polyQ-induced neurodegeneration correlated well with the degrees of loss-of-function, but appeared not to result from the inhibition of ex-polyQ aggregate formation. In the ex-polyQ-expressing cells of the late pupa, an upregulation of ter94 expression was observed prior to cell death. Coexpression of ter94 with ex-polyQ severely enhanced eye degeneration. Interestingly, when ter 94 was overexpressed in the eye by increasing the transgene copies, severe eye degeneration was induced. Furthermore, genetical studies revealed that ter94 was not involved in grim-, reaper-, hid-, ced4-, or p53-induced cell death pathways. From these observations, we propose that VCP is a novel cell death effector molecule in ex-polyQ-induced neurodegeneration, where the amount of VCP is critical. Control of VCP expression may thus be a potential therapeutic target in ex-polyQ-induced neurodegeneration.

Cell Death and Differentiation (2002) 9, 264-273. DOI: 10.1038/ sj/cdd/4400955
\end{abstract}

Keywords: ter94/VCP; polyglutamine; misfold protein; protein accumulation; neurodegeneration
Abbreviations: Ex-polyQ, expanded polyglutamine; TBP, tatabinding factor; $\mathrm{CBP}$, creb-binding protein; $\mathrm{N}-\mathrm{CoR}$, nuclear receptor corepressor; SRC-1, steroid receptor co-activator-1

\section{Introduction}

A growing number of inherited neurodegenerative diseases have been found to result from the expansion of unstable CAG trinucleotide repeats. ${ }^{1-3}$ To date, eight inherited neurodegenerative disorders have been recognized as belonging to this class of diseases. In each case, there is an expansion in the stretch of CAG repeats located in the coding region of the disease gene, resulting in an expanded polyglutamine (ex-polyQ) repeat in the diseased protein. Several lines of evidence have suggested that expansion of the poly $Q$ repeat confers a toxic gain-of-function property on the protein. This toxic property is correlated with an increased propensity for the diseased protein to misfold and form aggregates. Therefore, misfolding or altered solubility of expolyQ protein is thought to be a fundamental defect underlying the polyQ diseases. The existence of an ubiquitinated form of polyQ-containing aggregates associated with proteasome components indicates that neurons are attempting to reduce these abnormal protein aggregates, and that the elongation of the polyQ stretch may render the proteins to be resistant to proteasome-mediated degradation (for review, see ${ }^{4}$ ). Although ex-polyQ-containing aggregates or inclusions have been found in the nucleus of affected neurons from patients, animal models, and in cultured cell models, the pathological significance of these ex-polyQ aggregates is still under controversy. ${ }^{5,6}$

On the other hand, recent lines of evidence have indicated that ex-polyQ directly interact and co-localize with transcriptional factors, including TATA-binding factor (TBP), CREB-binding protein (CBP), p53, eye-absent, nuclear receptor co-repressor (N-CoR), mSin3A, CA150, steroid receptor co-activator-1 (SRC-1), and TAFII130. ${ }^{7-14}$ These results raise the possibility that dysregulation of these pathways through protein-protein interaction might cause a neuronal cell dysfunction seen in polyQ diseases.

Until now, several laboratories have reported the establishment of ex-polyQ-induced cell death in the Drosophila compound eye. ${ }^{15-18}$ These studies showed that eye degenerative phenotypes observed are ex-polyQ repeat length- and gene dosage-dependent, and were progressive with aging. These pathological features are similar to that of human polyglutamine diseases. Moreover, histochemical analyses revealed that ex-polyQ containing protein aggregates were observed as nuclear and cytoplasmic inclusions followed by cell death, namely eye degeneration.

Using Drosophila models, several groups identified that overexpression of molecular chaperones, including HSP70, 
HSP40 and dTPR2, can mitigate the ex-polyQ-induced eye degeneration without altering the ex-polyQ aggregates. ${ }^{19-21}$ This suppressive effect by molecular chaperones in the Drosophila system is consistent with the results of in vitro studies of mammalian models. ${ }^{22}$ Furthermore, Boates and colleagues performed large-scale genetical screening using SCA1 model flies and showed that mutants of transcriptional regulators such as $\operatorname{Sin} 3 A$, Rpd3, dCtBP and dSir2 modified the eye degeneration phenotypes. ${ }^{18}$ These lines of evidence further supported the notion that protein misfolding and subsequent abnormal protein interactions, especially between ex-polyQ and transcriptional factors, may cause transcriptional dysregulation leading to eye degeneration or cell death in Drosophila.

In this study, we performed a dominant modifier screen for enhancers and suppressors of rough eye phenotype caused by ex-polyQ expression in the developing eye. We screened through a combination of chromosomal deficients and a collection of P-element insertions, and identified several genes that alter the eye ablation phenotype. Here, we report the identification of the ter94 gene as one of the effector genes in the ex-polyQ-induced cell death pathway.

\section{Results}

\section{Establishment of a fly model of polyQ diseases}

Transgenes encoding an epitope-tagged 22, 79 and 92 glutamine repeat (Q22, Q79 and Q92, respectively) were designed and expressed in the compound eyes of Drosophila, by putting transgene expression under the control of the eye-specific glass promoter ( $p G M R)$. Like other models, ${ }^{15-18}$ we were able to successfully create fly models exhibiting degenerative eye phenotypes. The degenerative phenotypes observed were polyQ lengthand transgene dosage-dependent (Figure $1 \mathrm{~A}-\mathrm{C}$ ), and were progressive with aging (not shown). The formation of ex-polyQ aggregates, followed by severe cell death, was observed as seen in other models. ${ }^{15-18}$

\section{Intergenerational change of CAG trinucleotide repeats in Drosophila}

Upon maintaining the transgenic flies as homozygous stocks for several generations, a small population of flies was found to exhibit either a more severe or a milder eye phenotype than the parental flies (Figure 1D-F). The CAG repeat lengths in the transgenes of such flies were examined, since CAG repeat length is known to be the major determinant of the severity of degeneration, and moreover, the instability of expanded CAG repeats has been reported in several organisms, including humans, mice, E. coli, and yeast. ${ }^{23}$ Among the offspring of the GMR-Q79 homozygote flies, a further expansion of CAG repeat length was observed in less than $0.5 \%$ of the $F 1$ flies; further expanded CAG alleles of $92,83,81$ and 80 repeats were found in three independent $G M R-Q 79$ lines. On the other hand, approximately $3 \%$ of the $F 1$ flies exhibited a shortening of repeat length; shortened CAG alleles of $58,67,64,72$ and 76 repeats were found in the same three independent $G M R-Q 79$ lines. Simultaneous changes in CAG repeat lengths in both alleles were not observed. The degree of eye degeneration was highly sensitive to changes in repeat length, even though the change of repeat length occurred in only one transgenic allele (Figure 1D-F). Such intergenerational repeat instability was not observed in the four independent GMR-Q22 homozygous lines that were analyzed.

\section{Loss-of-function mutation in the ter94 gene dominantly suppresses polyQ-induced cell death phenotypes}

In order to identify genes involved in the polyQ-induced eye degeneration pathway, genetic screening was performed to search for dominant suppressors and enhancers of the rough eye phenotype. Namely, rough eye flies were crossed with 180 fly lines from a chromosomal deficiencybearing stock. Our survey revealed that one chromosomal deficient fly line, $D f(2 \mathrm{R}) \times 1$, which lacks the polytene chromosome bands 46C03-46E02, acts as a dominant suppressor. To identify the gene responsible for this suppression, available P-element inserted mutants with insertions mapping to the chromosomal position around the region absent in $D f(2 \mathrm{R}) \mathrm{X} 1$ were then tested. Through $\mathrm{P}$ element-orientated screening, two independent P-elementinserted lethal alleles, I(2)k15502 and I(2)03775 were found to act as weak dominant suppressors. Abnormalities in external eye structure, such as the fusion of ommatidia and loss of pigmentation, were improved by these P-element insertions (Figure 2A,B). The genomic DNA surrounding these insertions was isolated by plasmid rescue. Comparison of the sequences obtained with the Berkeley Drosophila genome project database (http://www.fruitfly.org/blast) revealed that both $\mathrm{P}$-elements were inserted in the $5^{\prime}$-noncoding region of the ter94 gene. Both of the Pelement inserted mutations caused zygotic embryonic lethality when homozygotic, and have been reported to be hypomorphic alleles. Ter94, the Drosophila homolog of $\mathrm{VCP} / \mathrm{p97/CDC48}$ and a member of the AAA+ class of the ATPase protein family, is 799 amino acids in length (approximately $94 \mathrm{kD}$ ), and contains two characteristic Walker motifs. $^{24}$

To confirm whether the ter94 loss-of-function mutation is responsible for the suppression of ex-polyQ-induced degeneration, the rough eye phenotypes of EMS-induced ter94 mutants were examined. ${ }^{25}$ In flies harboring a strong loss-offunction mutation of ter94 in one allele (ter9 $\left.4^{26-8} /+\right)$, the rough eye phenotype was dramatically suppressed (Figure $2 A, C, E-G)$. In flies harboring a reportedly weak loss-offunction mutation of ter94 in one allele $\left(\right.$ ter9 $\left.94^{22-26} /+\right)$, the rough eye phenotype was also suppressed, but the effect was weaker than that of ter9 $26-8 /+$ (Figure 2A,D). These results indicate that ter94 function is directly related to expolyQ-mediated eye degeneration. Furthermore, the identification of loss-of-function ter94 mutants as dominant suppressors implicates that ter94 plays an important role in the ex-polyQ-induced cell death pathway, most likely as an effector or a positive regulator. 

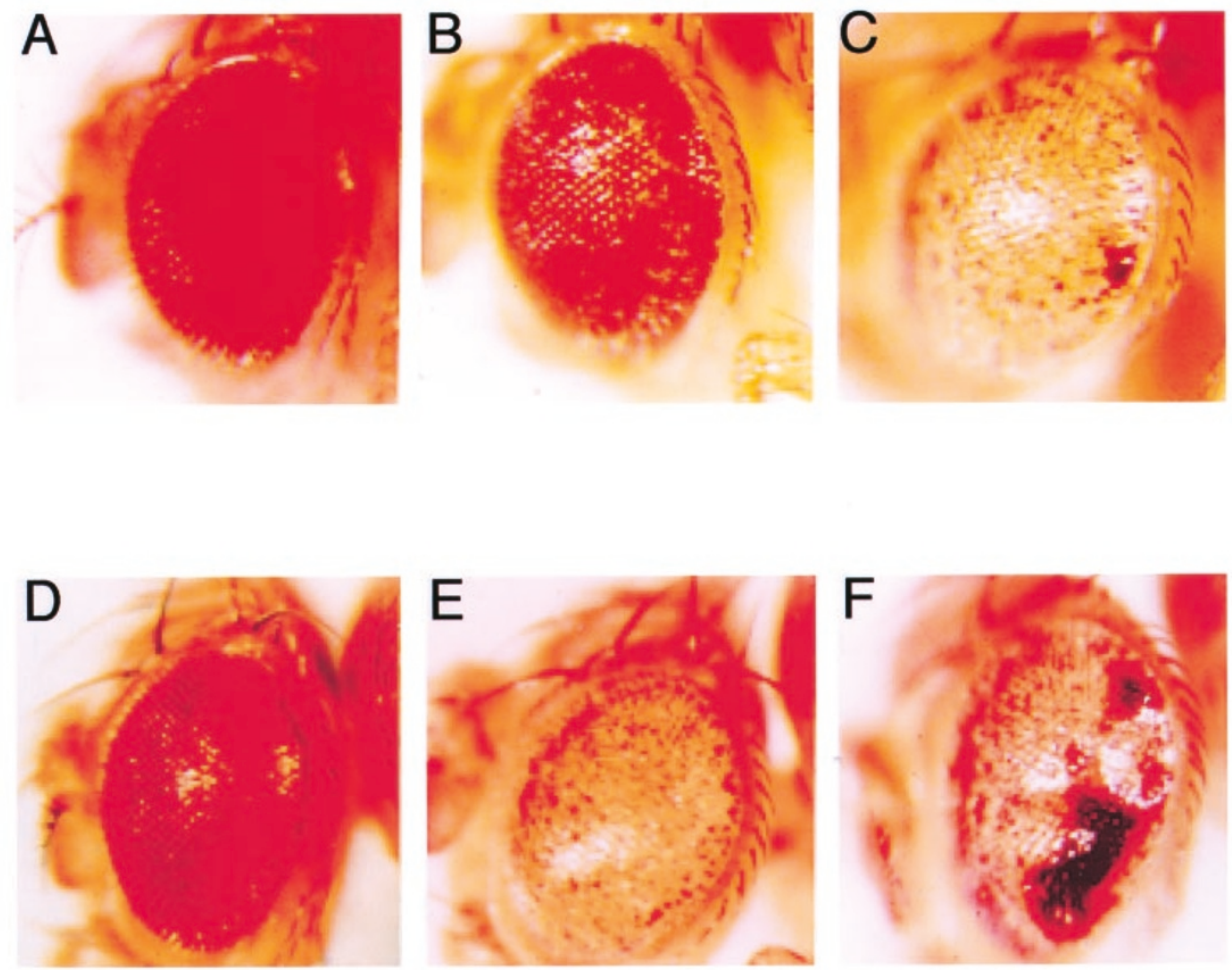

Figure 1 External eye phenotypes of polyQ-expressing flies. (A-C) Light photomicrographs of compound eyes from flies with the following genotypes are shown: (A) GMR-Q22/GMR-Q22, (B) GMR-Q79/+ and (C) GMR-Q79/GMR-Q79. (B,C) Loss of pigmentation and slight roughness of the external eyes are characteristic of the eye degeneration phenotype. (D-F) Intergenerational changes of expanded CAG repeats and the correlated rough eye phenotypes in Drosophila. Light photomicrographs of compound eyes from flies with the following genotypes are shown: (D) GMR-Q58/GMR-Q79, (E) GMR-Q79/GMR-Q79, and (F) GMRQ92/GMR-Q79. (F) Large necrotic spots appeared in the GMR-Q92/GMR-Q79 flies. CAG repeat numbers were determined by nucleotide sequencing of the PCR products
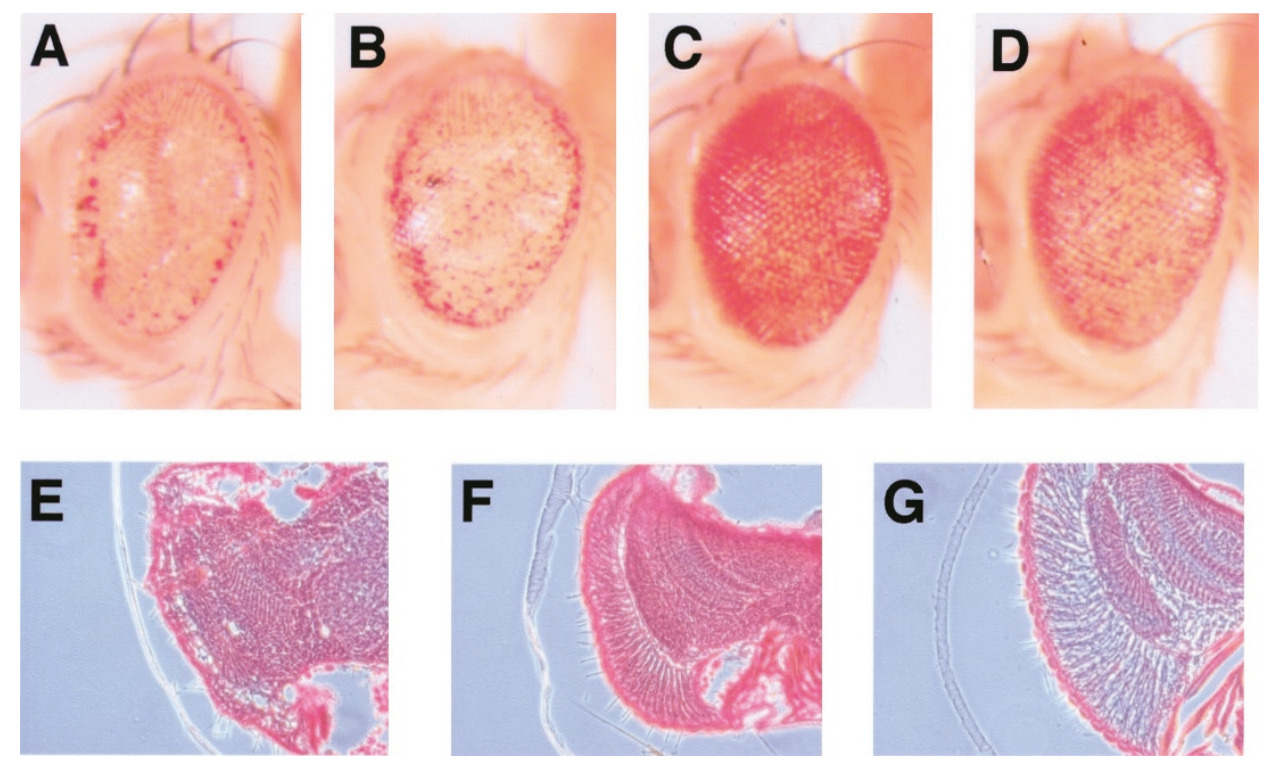

Figure 2 Dominant suppression of ex-polyQ-induced eye degenerations by loss-of-function mutations of the terg4 gene. (A-D) Light photomicrographs of the compound eye of (A) GMR-Q92/+, (B) GMR-Q92/ter94 $4^{\prime(2) 03775}$, (C) GMR-Q92/ter9426-8 and (D) GMR-Q92/ter94 $22-26$ flies are shown. (C) Pigmentation and smoothness of external eye surface were dramatically restored in the ter94 $26-8 /+$ background. (B) A lesser degree of restoration was also observed in the

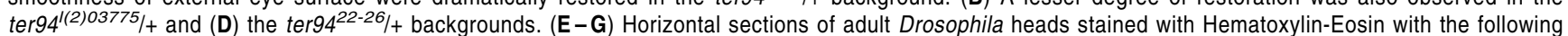
genotypes are shown: (E) GMR-Q92/+, (F) GMR-Q92/ter94 ${ }^{26-8}$, and (G) GMR-GAL4/+. (E) In GMR-Q92/+ flies, the internal structure of the compound eye was vigorously disrupted and most eye cells were shrunken or flattened. (F) In GMR-Q92/ter94 ${ }^{26-8}$ flies, internal eye structure and morphology of cells were dramatically restored. (G) In the control GMR-GAL4/+ flies, internal eye structure was normal 


\section{Genetical suppression of ex-polyQ-induced neurodegeneration by ter 94 loss-of-function mutations is not a result of the inhibition of ex- poly $Q$ aggregate formation}

To date, the issue as to whether the formation of ex-polyQ aggregates is causative for the neurodegeneration seen in human polyQ diseases or not has been under controversy. Hence, in order to test whether the dominant suppression of eye degeneration by the loss-of-function of ter94 is accompanied by a change in ex-polyQ aggregates, the level of expolyQ aggregate formation in the eye discs from third instar larvae and the compound eye of pupa were immunohistochemically analyzed using anti-epitope antibodies. In the retina of flies harboring one copy of GMR-Q92 and ter94 ${ }^{26-8}$ on one allele, the total amounts and sizes of the ex-polyQ aggregates were almost the same as that of control flies carrying just one copy of $G M R-Q 92$ (Figure $3 \mathrm{~A}, \mathrm{~B}$ ). Regarding subcellular localization and the time of onset of aggregation, no clear difference between the two lines could be found. The eye discs of late pupa were also analyzed, but no clear difference could be observed in the formation of aggregates at this stage either (Figure 3C,D). These results indicate that the dominant suppression of ex-polyQ-induced neurodegeneration by ter94 loss-of-function mutations is not a result of the inhibition of ex-polyQ aggregate formation.

\section{The expression of ter94 is upregulated at a relatively late stage in the ex-polyQ expressing cells}

In order to examine ter94 protein expression and to determine its localization, the compound eye of late pupa were stained using affinity purified anti-ter94 polyclonal serum. Immunohistochemical analyses revealed intense signals in the retina of the GMR-Q92 lines (Figure 4A-C). In contrast to the GMR$Q 92$ lines, only faint signals were observed in the control GMR-GAL4 lines (Figure 4D-F). In the GMR-Q92 lines,
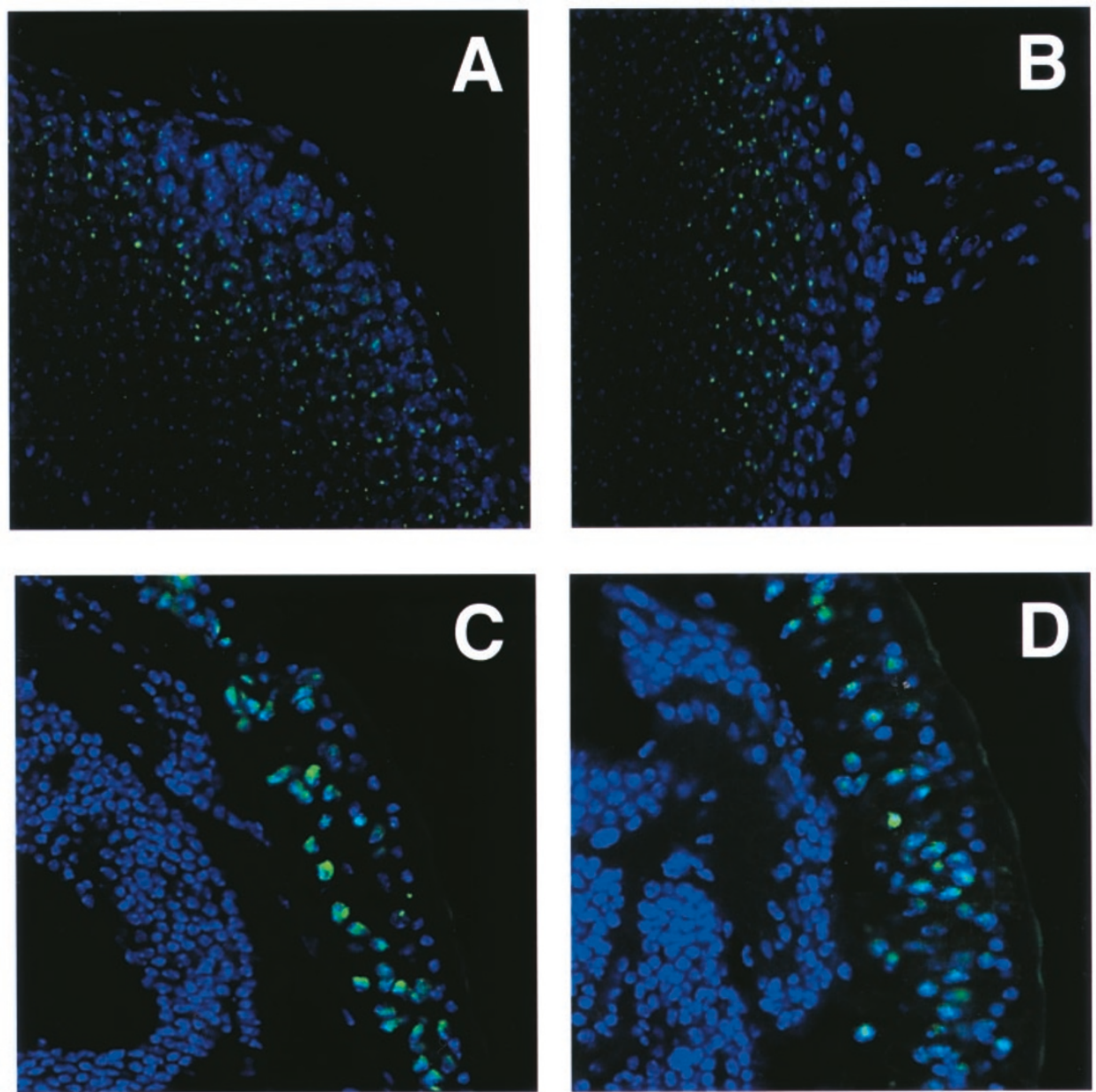

Figure 3 Suppression of ex-polyQ-induced eye degeneration by loss-of-function ter94 mutations without the inhibition of ex-polyQ-aggregate formation. (A,B) Confocal microscopy images of the eye imaginal discs from third instar larvae and $(\mathbf{C}, \mathbf{D})$ retina from late pupa with the following genotypes are shown: $(\mathbf{A}, \mathbf{C})$ GMRQ92/+ and (B,D) GMR-Q92/ter94 ${ }^{26-8}$. Ex-polyQ was detected with anti-FLAG antibody (Green). The nuclei were stained with DAPI (Blue). (A,B) There was no difference in the amount and localization of ex-polyQ aggregates between the wildtype and the ter94 ${ }^{26-8}$ background in the eye discs of late third instar larvae. $(C, D)$ There was no difference in expression levels and localization of ex-polyQ-aggregates between the wildtype and the $\operatorname{ter} 94^{26-8} /+$ background in the late pupa stage, although the histological organization of retina was improved in the $t e r 94^{26-8} /+$ background 

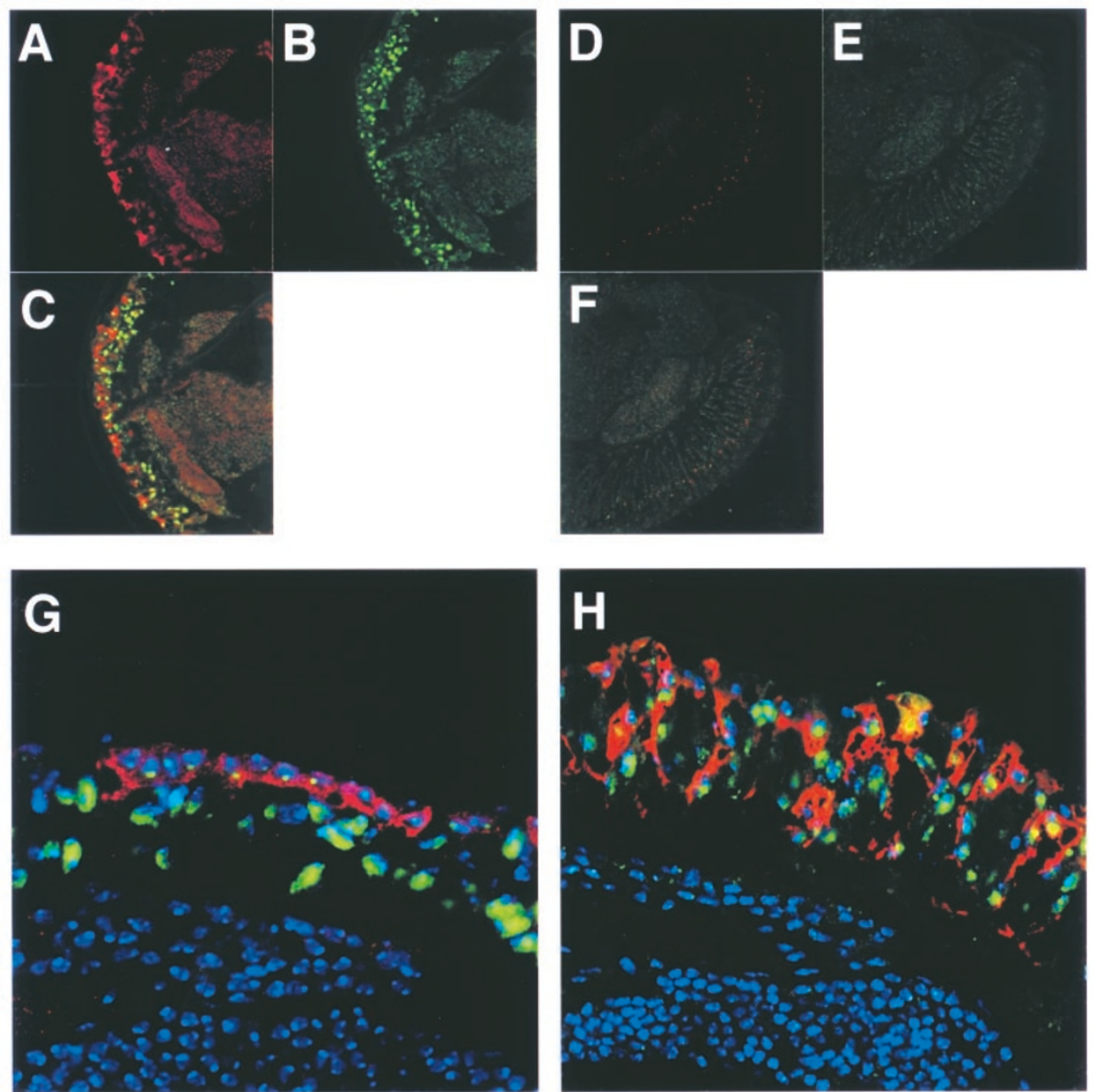

Figure 4 Expression of ter94 protein was upregulated in the ex-polyQ-expressing eyes. (A-H) Horizontal sections of adult Drosophila heads were stained with anti-ter94 polyclonal antibody (Red), anti-FLAG antibody (Green) for detecting ex-polyQ (Q92), and DAPI (Blue) for visualizing cell nuclei. Confocal microscopic images of retina from the following genotypes are shown: $(\mathbf{A}-\mathbf{C}, \mathbf{G})$ GMR-Q92/+, (D-F) GMR-GAL4/+, and (H) GMR-Q92/terg4 ${ }^{26-8}$. Overlay images of ter94 and ex-polyQ $(\mathbf{C}, \mathbf{F})$ and all three markers $(\mathbf{G}, \mathbf{H})$ are also presented

ter94-positive signals were mainly detected diffusely in the cytoplasm of cells with ex-polyQ aggregates (Figure $4 \mathrm{G}$ ), but some ter94 signals were co-localized with ex-polyQ aggregates (Figure 4C). These strongly ter94-positive cells were morphologically flattened. On the other hand, in the retina of transgenic flies harboring ter $94^{26-8} /+$, while an enhanced ter94 signal was also observed, the morphology of the compound eye was relatively retained (Figures $2 \mathrm{~F}$ and $4 \mathrm{H}$ ). In order to further analyze ter94 expression at an earlier stage, the eye discs of third instar larvae were examined. In the eye discs from the GMR-Q92 lines, the expression of ter94 was faint in the cytoplasm, and no difference was observed when compared with the wildtype (data not shown). These results suggest that the expression of ter94 is upregulated at a relatively late stage even in the presence of ex-polyQ expression.

\section{Co-expression of ter94 with ex-polyQ severely enhances eye degeneration}

In order to address whether an elevated level of ter94 alters ex-polyQ-induced eye degeneration, ter94 transgenic flies (GMR-GAL4;UAS-ter94) were generated and crossed with ex-polyQ-expressing lines. Co-expression of ter94 with Q92 or with Q79 both led to enhancement of rough eye phenotypes in these flies (Figure $5 A-G$ ). With this genetic background, the extent of eye roughness induced by one copy of ex-polyQ increased to a level almost equal to that induced by two copies of ex-polyQ in the normal genetic background (Figure 5A-F). The amount and size of ex-polyQ aggregates did not recognizably change upon co-expression of ter94, although much more co-localization of ex-polyQ and ter94 was observed (Figure $5 \mathrm{G}, \mathrm{H})$.

\section{Overexpression of ter94 induces apoptotic cell death}

Flies carrying GMR-GAL4;UAS-ter94 exhibited a mild eye ablation phenotype characterized by a slight loss of red pigmentations, irregular ommatidial packing and missing inter ommatidial bristles (Figure 6A,C). When the transgene was increased to two copies (GMR-GA- 

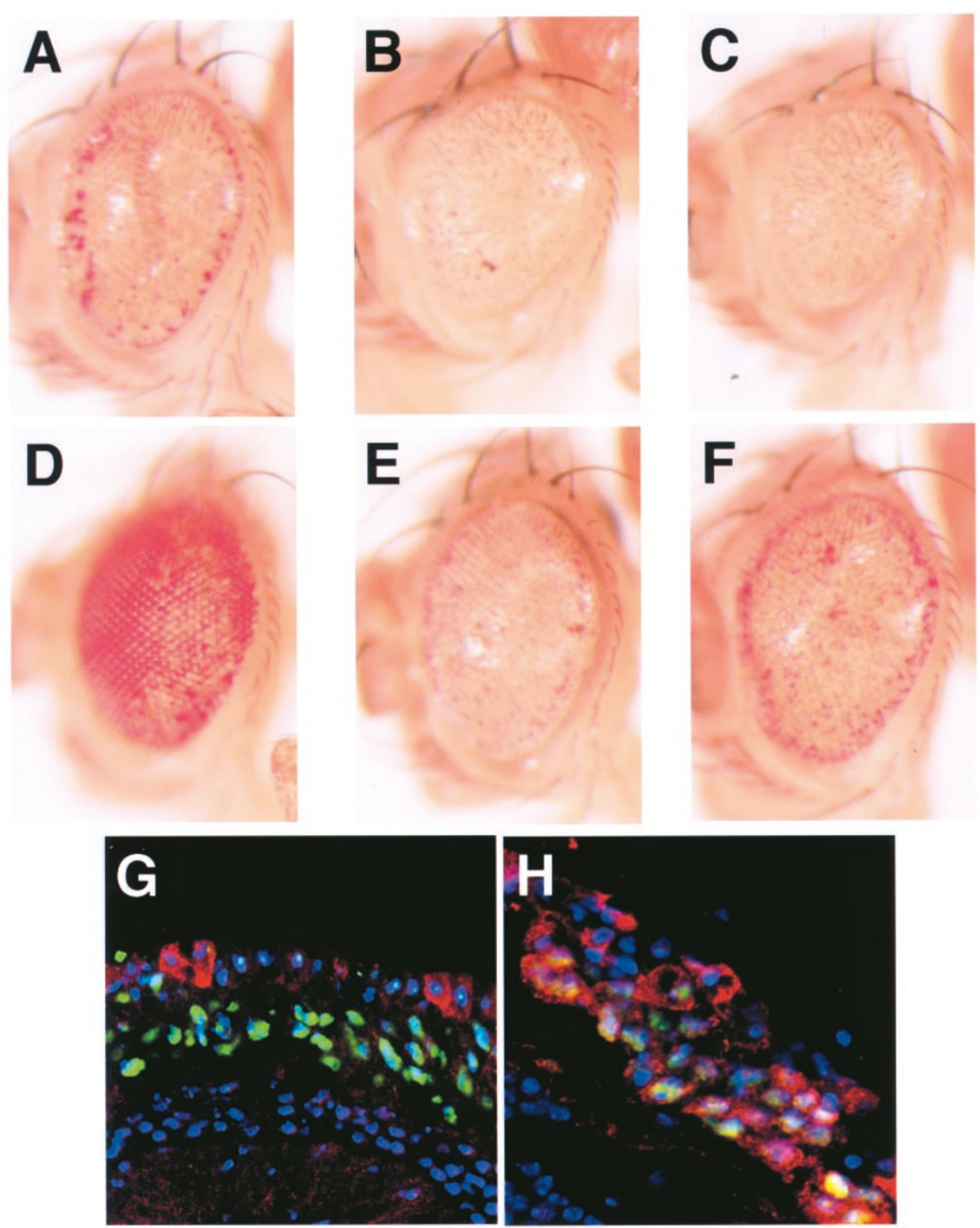

Figure 5 Enhancement of ex-polyQ-induced eye degeneration by the additional expression of ter94. (A-F) Light photomicrographs of adult Drosophila compound eye with the following genotypes are shown: (A) GMR-Q92/+, (B) GMR-GAL4/+;GMR-Q92/UAS-ter94, (C) GMR-Q92/GMR-Q92, (D) GMRQ79/+, (E) GMR-GAL4/+;GMR-Q79/UAS-ter94 and (F) GMR-Q79/GMR-Q79. Excess expression of ter94 by the transgene enhanced the external eye roughness and loss of pigmentation in flies harboring (B) one copy of GMR-Q92 and (E) GMR-Q79, which were comparable to flies carrying (C) two copies of GMR-Q92 and (F) GMR-Q79, respectively. $(\mathbf{G}, \mathbf{H})$ Immunohistochemical detection of ex-polyQ aggregates and ter94 in the Drosophila compound eye in the (G) GMR-Q92/+ and (H) GMR-GAL4/+;GMR-Q92/UAS - ter94 genotypes. Samples were stained with anti-ter94 polyclonal antibody (Red), anti-FLAG antibody (Green) for detecting ex-polyQ (Q92), and DAPI (Blue) for visualizing cell nuclei. Confocal images of all three markers are overlaid and presented

L4;UAS-ter94/UAS-ter94), eye tissue became considerably degenerated and the size of compound eye became extremely small (Figure 6B,D). The external eye surface appeared to be less pigmented and glassy (Figure 6B). TUNEL analysis of head sections revealed that massive apoptotic cell death has occurred in the compound eye cells (Figure $6 \mathrm{E}-\mathrm{G}$ ), suggesting that ter94 itself has an apoptosis-inducing activity and the upregulation of ter94 seen in polyQ-expressing cells are a direct cause of neuronal cell death.

\section{Ter94-induced cell death pathway proceeds via a different pathway from known cell death pathway}

In Drosophila, three major apoptosis-inducing genes, grim, reaper, and hid have been isolated. ${ }^{26-28}$ Overexpression of each of these three genes, as well as ced 4 and human $p 53$ in the compound eyes of Drosophila led to eye degenerative phenotypes to different degrees (Figure 7). ${ }^{29,30}$ Genetic analyses were performed to examine whether ter94 is involved in the cell death pathway used by these apoptosis 

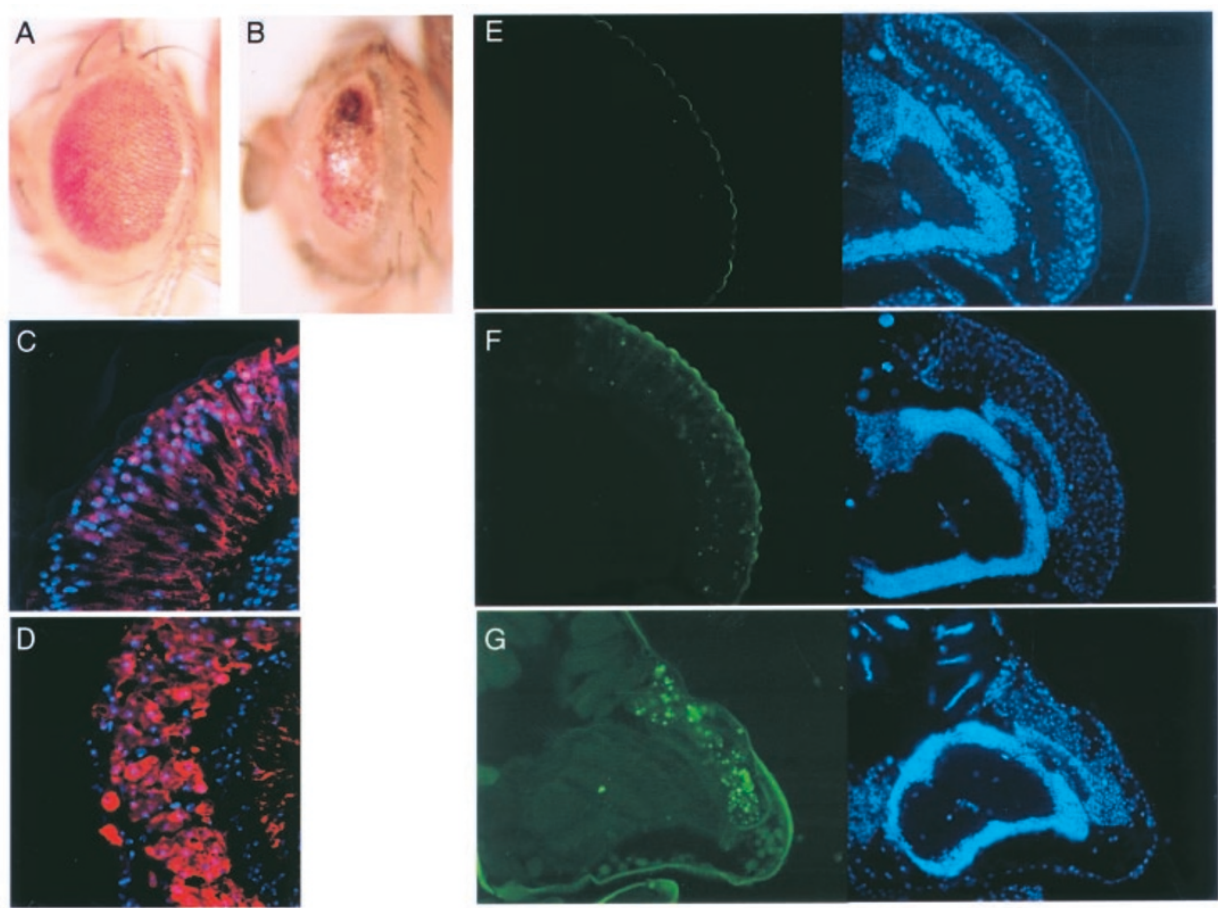

Figure 6 Over expression of ter94 protein caused the dosage-dependent cell death in Drosophila compound eye. (A,B) Light photomicrographs of adult Drosophila compound eye in the (A) GMR-GAL4/+;UAS-ter94/+ and (B) GMR-GAL4/+;UAS -ter94/UAS-ter94 genotypes are shown. Immunohistochemical detection of ter94 in the Drosophila compound eye in the (C) GMR-GAL4/+;UAS-ter94/+ and (D) GMR-GAL4/+;UAS-ter94/UAS-ter94 genotypes. Samples were stained with anti-ter94 polyclonal antibody (Red) and DAPI (Blue) for visualizing cell nuclei. (E-G) The horizontal sections of late pupa heads were stained by the TUNEL method (Green) and DAPI (Blue). Genotypes; (E) GMR-GAL4/GMR-GAL4, (F) GMR-GAL4/+; UAS-ter94/+ and (G) GMR-GAL4/+;UAS-ter94/ UAS-terg4

inducers. Namely, flies carrying GMR-grim, GMR-reaper, GMR-hid, GMR-ced4, or GMR-GAL4;UAS-p53 were crossed with terg $4^{26-8} /+$. In contrast to ex-polyQ-induced eye degeneration, the ter $94^{26-8} /+$ background did not cause inhibition of the eye degenerations induced by these cell death inducers (Figure 7). These results clearly indicate that ter94 does not function as an effector of cell death by acting downstream of these cell death inducers, and thus specifies the effector function of ter94 in ex-polyQ-induced cell death.

\section{Discussion}

In an attempt to screen for the effector molecule in ex-polyQ cell death pathway, we performed genetical F1 screening of dominant suppressor and enhancers. As a result of the screening, we identified loss-of-function mutants of ter94, Drosophila VCP/p97, as a dominant suppressor. In ex-polyQ expressing cells, ter94 expression was significantly upregulated prior to cell death. Furthermore, transgenic fly study revealed that the overexpression of ter94 induced severe apoptotic cell death in the compound eye. These results suggest that ter94 seems to play a key role as an effector in ex-polyQ cell death pathway.

VCP is ubiquitously expressed and highly conserved between various species; BLAST analyses revealed that ter94 is $84 \%$ identical to the human VCP/p97 and $67 \%$ identical to yeast CDC48p. Members of the AAA+ class of ATPase have been reported to be involved in a variety of biological processes, including protein degradation. ${ }^{31,32}$ Biochemical and genetic analyses have shown that ter94 is involved in fusome formation ${ }^{33}$ and oskar mRNA localization to the Drosophila egg chamber. ${ }^{25}$ There have been no previous reports stating the involvement of VCP in neurodegeneration, although a possible link between VCP and cell death in other situations has been reported. ${ }^{34,35}$ For other purposes, we biochemically purified a protein interacting with the MJD protein containing an ex-polyQ from mammalian cells, which turned out to be VCP. ${ }^{36}$ Furthermore, we observed that the expression of certain VCP mutants in cultured mammalian cells induce cell death. ${ }^{36}$

In dividing cells, VCP/CDC48 has been reported to perform multiple roles in cell cycle progression, such as during membrane fusion, ${ }^{37}$ spindle body/centrosome duplication, ${ }^{38}$ organelle assembly, ${ }^{39}$ etc. Through independent genetical (this study) and cell biological analyses, ${ }^{36}$ we found evidence that VCP/ter94 can function as a cell death inducer or effector in certain conditions. These apparently opposing effects of VCP/ter94 on cell cycle progression and cell death are confusing. However, the complexity of the various functions of VCP/ter94 could possibly be due to differences in cell states, e.g. cells in the dividing state and in the post-mitotic state. The ex-polyQ-expressing cells in our fly system included photoreceptor neurons, cone cells, and pigment cells, which are all nondividing cells. Consistent with this, ex-polyQ-mediated cell death was not clearly observed in the dividing imaginal disc cells. ${ }^{15}$ 


\section{grim}

\section{2 copies}

1 copy
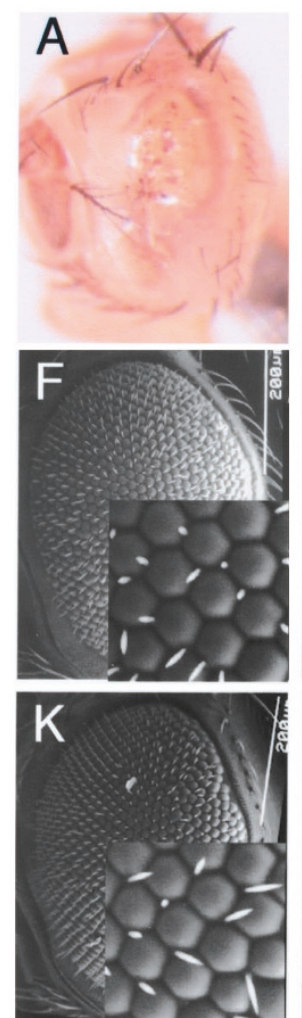
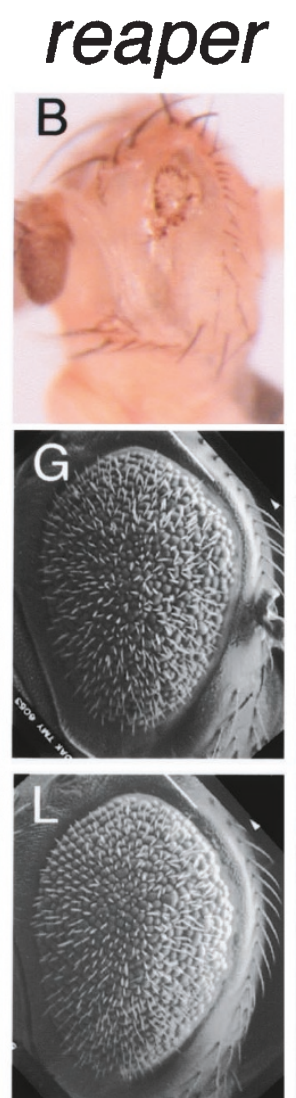

hid
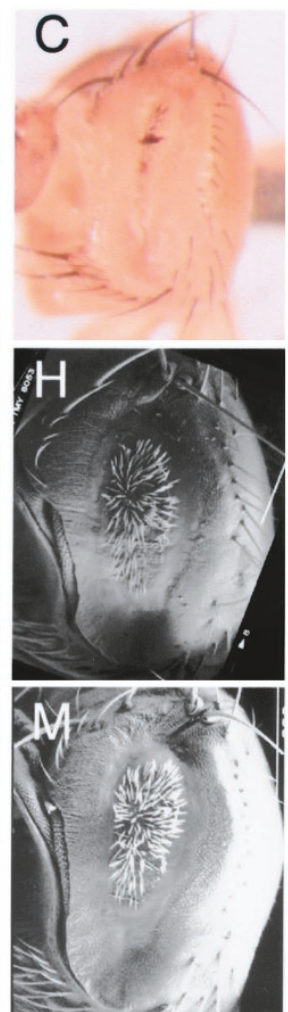

ced4
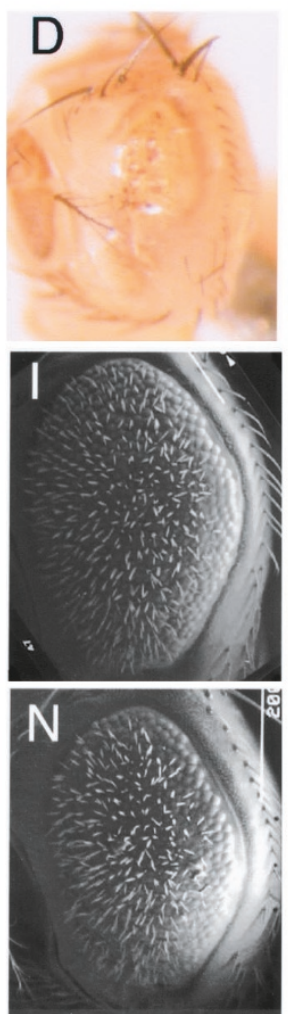

p53
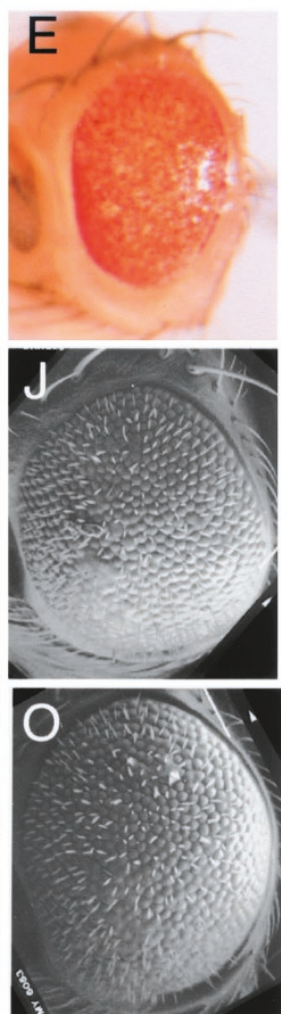

Figure 7 The lack of involvement of ter94 in grim-, reaper-, hid-, ced4, and p53-mediated cell death pathways. (A-E) Light and (F-O) electron photomicrographs of compound eyes of transgenic flies with the following genotypes are shown: (A) GMR-grim1/GMR-grim1, (B) GMR-reaper46/GMR-reaper46, (C) GMRhid10/GMR-hid10, (D) GMR-ced4/GMR-ced4, (E) GMR-GAL4/+;UAS-p53/UAS-p53, (F) GMR-grim1/+, (G) GMR-reaper46/+, (H) GMR-hid10/+, (I) GMR-ced4/+, (J) GMR-GAL4/+;UAS-p53/+, (K) GMR-grim1/ter94 $4^{26-8}$, (L) ter94 26-8/+; GMR-reaper46/+, (M) GMR-hid10/ter94 ${ }^{26-8}$, (N) GMR-ced4/ter94 ${ }^{26-8}$ and (O) GMR - GAL4/+;UAS - p53/ter94 26-8. (A-J) Expression of each of the cell death-inducing genes in the Drosophila compound eye exhibited different degrees of eye degeneration phenotypes in a gene-dosage dependent manner. $(K-0)$ Note that these eye degeneration phenotypes were not rescued at all in the terg4 $26-8 /+$ background

Indeed, the selective loss or cell death of post-mitotic neurons is the prominent feature in many human neurodegenerative disorders.

One characteristic feature of polyQ-induced neurodegeneration is late onset, even in animal models. In Drosophila polyQ disease models including our flies, the formation of ex-polyQ aggregates was found in the third instar larvae, but ex-polyQ-induced cell death begins mainly in the late pupa stage. This time period between the third instar larvae stage and late pupa stage is indeed the period that was found to exhibit an increase in ter94 expression. These observations lead to the idea that both the amount and timing of ter94expression are crucial to its effector function in ex-polyQinduced cell death. This idea is further supported by our transgenic fly experiments of ter94, where elevated ter94 levels were found to induce severe apoptotic cell death. Genetical interaction experiments using Drosophila apoptosis inducing genes implicated the ter94-mediated pathway was different from these pathways. Further genetic studies will contribute to understanding the mechanism involved in ter94-mediated cell death pathway.
Recent genetic studies using the Drosophila system have shown that overexpression of a number of molecular chaperones such as HSP70, HSP40 and dTPR2 can mitigate the ex-polyQ-induced eye degeneration phenotype without altering the ex-polyQ aggregate. ${ }^{19-21}$ The mechanisms leading to such inhibition, however, remain controversial. It is noteworthy that we have identified physical interactions between VCP and both HSP70 and HSP40 by biochemical analyses (unpublished observations). Overexpression of these chaperones may mask VCP/ter94, thus inhibiting its effector function in ex-polyQ induced eye degeneration, a possibility that remains to be tested. We have also isolated several other mutant fly lines, including those with mutations in several transcription factors, which dominantly modify the ex-polyQ-induced eye degenerations. Further analyses of these mutants, especially with regard to their roles in the control of VCP expression and as potential genes acting downstream of ter94 in the cell death pathway, will shed further light on the understanding of the molecular basis of the polyQ diseases. 


\section{Materials and Methods}

\section{Drosophila strains and genetics}

Fly culture and crosses were performed at $25^{\circ} \mathrm{C}$ on standard food. We screened 180 lines carrying chromosomal deficiency for an enhancement or a suppression of the rough eye phenotypes induced by ex-polyQ expression. Chromosomal deficiency fly lines were supplied by Dr. S Hayashi (National Institute of Genetics, Japan). Two P-element insertions, I/(2)k15502 and I(2)03775 were obtained from the Drosophila Stock Center at Bloomington, Indiana, USA. Dr. ES Goldstein (Arizona State University, USA) kindly provided flies carrying EMS-induced lethal alleles of ter94. Dr. H Steller (Massachusetts Institute of Technology, USA) kindly provided GMR-reaper46 and GMR-hid10. Dr. JM Abrams (University of Texas Southwestern Medical Center, USA) kindly provided GMRgrim1. Dr. M Miura (Osaka University, Japan) kindly provided GMRced-4. We sincerely thank these scientists for their valuable materials.

\section{Construction of transgene and Plasmid rescue}

To generate the poly CAG tracts, genomic DNA from MachadoJoseph Disease patient containing 79 and 92 repeat of CAG and normal control were used as a template for PCR synthesis. ${ }^{40}$ Resulting PCR fragments were digested with $\mathrm{BamHI}$ and $E c \mathrm{RI}$ and inserted in-frame to a FLAG tag sequence into BamHI-EcoRI fragment of $\mathrm{PCMX}-\mathrm{FLAG}$ vector. ${ }^{41}$ HindllI - EcoRI fragments containing FLAG tag and CAG repeat were blunted with Klenow Fragment (Roche) and subcloned into Stul site of pGMR vector. ${ }^{42}$ A ter94 cDNA fragment that included the entire coding region as well as $110 \mathrm{bp}$ of 5 untranslated sequence and $560 \mathrm{bp}$ of $3^{\prime}$ untranslated sequence was cloned into the pUAS vector. Subcloning, mapping and DNA sequencing were performed by standard protocols. DNA flanking the insertion site was isolated following digestion of genomic DNA from I(2) k15502 and I(2)03775 with EcoRI and subsequent rescue of circularized plasmid. A $1.0 \mathrm{~kb}$ fragment from the rescued DNA were sequenced and sequence comparison was done with the BLAST server at BDGP. Sequence analysis was performed by dideoxy chain termination procedure using an $\mathrm{ABI} D N A$ sequencer.

\section{Generation of transgenic flies}

Transgenic flies were generated by microinjecting pGMR-Q79, pGMR-Q92, pGMR-Q22 and pUAS-ter94 according to standard protocols. Several independent lines were established from each transgene and their integration sites were mapped to individual chromosomes.

\section{Immunohistochemistry and TUNEL analysis}

Imaginal discs from third instar larvae were dissected in Drosophila's Ringer's solution and fixed in $4 \%$ paraformaldehyde (PFA)/PBS for $30 \mathrm{~min}$ at room temperature or at $4^{\circ} \mathrm{C}$. The heads of pupas were decapitated and fixed in $4 \%$ PFA/PBS for $12 \mathrm{~h}$ at $4{ }^{\circ} \mathrm{C}$. After fixation, heads were incubated in increasing concentrations of sucrose/PBS (from $10 \%$ to $30 \%$ ) at $4{ }^{\circ} \mathrm{C}$, and then embedded in OCT4583 (TissueTek), frozen on dry-ice, and sectioned. After washing in PBS $/ 0.3 \%$ TritonX-100 (PBT), the samples were blocked with PBT containing $10 \%$ normal goat serum for $30 \mathrm{~min}$ at room temperature and then incubated with a mouse anti-flag monoclonal antibody M5 (Sigma) at $1: 1000$ dilution and rabbit anti-ter 94 serum at $1: 500$ dilution at $4{ }^{\circ} \mathrm{C}$ for $12 \mathrm{~h}$. After extensive washing with PBT, samples were incubated with FITC-labeled goat anti-mouse or texas red-labeled anti-rabbit second antibody (Jackson Immunoresearch) at 1:2000 dilution for $3 \mathrm{~h}$ at $4^{\circ} \mathrm{C}$. After extensive washing with PBT, samples were mounted in VECTORSHIELD mounting medium with DAPI (Vector Laboratories) and were analyzed by confocal microscopy (Carl Zeiss). The TUNEL assay was performed using In Situ Cell Death Detection kit (Roche) following manufacturer's protocol.

\section{Generation of anti-ter94 polyclonal antibody}

Anti-ter94 rabbit polyclonal antibody was generated by standard methods using a full-length ter94 protein fused to glutathione-Stransferase (GST-ter94) as an antigen. GST-ter94 was purified by ion exchange chromatography and injected subcutaneously into rabbits for immunization. Affinity purification of the serum was performed using AminoLink Immobilization kit (Pierce).

\section{Scanning electron microscopy}

Adult flies were anesthetized, mounted and observed under a Hitachi S-100 scanning electron microscope in the low vacuum mode.

\section{Acknowledgments}

We thank H Yoshii, M Sugimoto and HA Popiel for secretarial assistance in the preparation of this manuscript and RT Y-Umesono for valuable advice and suggestions. This work was supported in part by research grants from the Ministry of Education, Science, Sports, and Technology of Japan, and the Ministry of Health, Labour, and Welfare of Japan, the Yamanouchi Foundation for Research on Metabolic Disorders, the Naito Foundation, and the Uehara Foundation.

\section{References}

1. Kakizuka A (1998) Protein precipitation: a common etiology in neurodegenerative disorders? Trends Genet. 14: 396-402

2. Paulson HL, Bonini NM and Roth KA (2000) Polyglutamine disease and neuronal cell death. Proc. Natl. Acad. Sci. USA 97: 12957-12958

3. Zoghbi HY and Orr HT (2000) Glutamine repeats and neurodegeneration. Annu. Rev. Neurosci. 23: 217-247

4. Paulson HL (1999) Protein fate in neurodegenerative proteinopathies: polyglutamine diseases join the (mis)fold. Am. J. Hum. Genet. 64: 339-345

5. Klement IA, Skinner PJ, Kaytor MD, Yi H, Hersch SM, Clark HB, Zoghbi HY and Orr HT (1998) Ataxin-1 nuclear localization and aggregation: role in polyglutamine-induced disease in SCA1 transgenic mice. Cell 95: 41-53

6. Saudou F, Finkbeiner S, Devys D and Greenberg ME (1998) Huntingtin acts in the nucleus to induce apoptosis but death does not correlate with the formation of intranuclear inclusions. Cell 95: 55-66

7. Perez MK, Paulson HL, Pendse SJ, Saionz SJ, Bonini NM and Pittman RN (1998) Recruitment and the role of nuclear localization in polyglutamine-mediated aggregation. J. Cell Biol. 143: 1457-1470

8. Boutell JM, Thomas P, Neal JW, Weston VJ, Duce J, Harper PS and Jones AL (1999) Aberrant interactions of transcriptional repressor proteins with the Huntington's disease gene product, huntingtin. Hum. Mol. Genet. 8: 1647-1655

9. Kazantsev A, Preisinger E, Dranovsky A, Goldgaber D and Housman D (1999) Insoluble detergent-resistant aggregates form between pathological and nonpathological lengths of polyglutamine in mammalian cells. Proc. Natl. Acad. Sci. USA 96: 11404-11409

10. Stenoien DL, Cummings CJ, Adams HP, Mancini MG, Patel K, DeMartino GN, Marcelli M, Weigel NL and Mancini MA (1999) Polyglutamine-expanded androgen receptors form aggregates that sequester heat shock proteins, proteasome components and SRC-1, and are suppressed by the HDJ-2 chaperone. Hum. Mol. Genet. 8: 731-741 
11. Shimohata T, Nakajima T, Yamada M, Uchida C, Onodera O, Naruse S, Kimura T, Koide R, Nozaki K, Sano Y, Ishiguro H, Sakoe K, Ooshima T, Sato A, Ikeuchi T, Oyake M, Sato T, Aoyagi Y, Hozumi I, Nagatsu T, Takiyama Y, Nishizawa M. Goto J, Kanazawa I, Davidson I, Tanese N, Takahashi H and Tsuji S (2000) Expanded polyglutamine stretches interact with TAFII130, interfering with CREBdependent transcription. Nat. Genet. 26: 29-36

12. Steffan JS, Kazantsev A, Spasic-Boskovic O, Greenwald M, Zhu YZ, Gohler H, Wanker EE, Bates GP, Housman DE and Thompson LM (2000) The Huntington's disease protein interacts with p53 and CREB-binding protein and represses transcription. Proc. Natl. Acad. Sci. USA 97: 6763-6768

13. Holbert S, Denghien I, Kiechle T, Rosenblatt A, Wellington C, Hayden MR, Margolis RL, Ross CA, Dausset J, Ferrante RJ and Neri C (2001) The Gln-Ala repeat transcriptional activator $\mathrm{CA} 150$ interacts with huntingtin: neuropathologic and genetic evidence for a role in Huntington's disease pathogenesis. Proc. Natl. Acad. Sci. USA 98: 1811-1816

14. Nucifora Jr FC, Sasaki M, Peters MF, Huang H, Cooper JK, Yamada M, Takahashi H, Tsuji S, Troncoso J, Dawson VL, Dawson TM and Ross CA (2001) Interference by huntingtin and atrophin-1 with CBP-mediated transcription leading to cellular toxicity. Science 291: 2423-2428

15. WarrickJM, Paulson HL, Gray-Board GL, Bui QT, Fischbeck KH, Pittman RN and Bonini NM (1998) Expanded polyglutamine protein forms nuclear inclusions and causes neural degeneration in Drosophila. Cell 93: 939-949

16. Jackson GR, Salecker I, Dong X, Yao X, Arnheim N, Faber PW, MacDonald ME and Zipursky SL (1998) Polyglutamine-expanded human huntingtin transgenes induce degeneration of Drosophila photoreceptor neurons. Neuron 21: 633642

17. Marsh JL, Walker H, Theisen H, Zhu YZ, Fielder T, Purcell J and Thompson LM (2000) Expanded polyglutamine peptides alone are intrinsically cytotoxic and cause neurodegeneration in Drosophila. Hum. Mol. Genet. 9: 13-25

18. Fernandez-Funez P, Nino-Rosales ML, de Gouyon B, She WC, Luchak JM, MartinezP, Turiegano E, BenitoJ, Capovilla M, SkinnerPJ, McCall A, Canall, Orr HT, Zoghbi HY and Botas J (2000) Identification of genes that modify ataxin-1induced neurodegeneration. Nature 408: 101-106

19. Warrick JM, Chan HY, Gray-Board GL, Chai Y, Paulson HL and Bonini NM (1999) Suppression of polyglutamine-mediated neurodegeneration in Drosophilaby the molecular chaperone HSP70. Nat. Genet. 23: 425-428

20. Chan HY, Warrick JM, Gray-Board GL, Paulson HL and Bonini NM (2000) Mechanisms of chaperone suppression of polyglutamine disease: selectivity, synergy and modulation of protein solubility in Drosophila. Hum. Mol. Genet. 9: $2811-2820$

21. Kazemi-Esfarjani $P$ and Benzer S (2000) Genetic suppression of polyglutamine toxicity in Drosophila. Science 287: 1837-1840

22. Cummings CJ, Mancini MA, Antalffy B, DeFranco DB, Orr HT and Zoghbi HY (1998) Chaperone suppression of aggregation and altered subcellular proteasome localization imply protein misfolding in SCA1. Nat. Genet. 19: $148-154$

23. Wells RD and Warren ST (1998) Genetic Instabilities and Hereditary Neurological Diseases. (Academic Press, SanDiego)

24. Pinter M, Jekely G, Szepesi RJ, Farkas A, Theopold U, Meyer HE, Lindholm D, Nassel DR, Hultmark D and Friedrich P (1998) TER94, a Drosophila homolog of the membrane fusion protein $C D C 48 / p 97$, is accumulated in nonproliferating cells: in the reproductive organs and in the brain of the imago. Insect Biochem. Mol. Biol. 28: $91-98$
25. Ruden DM, Sollars V, Wang X, Mori D, Alterman M and Lu X (2000) Membrane fusion proteins are required for oskar mRNA localization in the Drosophila egg chamber. Dev. Biol 218: 314-325

26. Chen P, Nordstrom W, Gish B and Abrams JM (1996) grim, a novel cell death gene in Drosophila. Genes \& Dev. 10: 1773-1182

27. White K, Grether ME, Abrams JM, Young L, Farrell Kand Steller H(1994) Genetic control of programmed cell death in Drosophila. Science 264: 677-683

28. Grether ME, Abrams JM, Agapite J, White K and Steller H (1995) The head involution defective gene of Drosophila melanogaster functions in programmed cell death. Genes \& Dev. 9: $1694-1708$

29. Kanuka H, Hisahara S, Sawamoto K, Shoji S, Okano H and Miura M (1999) Proapoptotic activity of Caenorhabditis elegans CED-4 protein in Drosophila: implicated mechanisms for caspase activation. Proc. Natl. Acad. Sci. USA 96: $145-150$

30. Yamaguchi M, Hirose F, Inoue YH, Shiraki M, Hayashi Y, Nishi Y and Matsukage A (1999) Ectopic expression of human 553 inhibits entry into Sphase and induces apoptosis in the Drosophila eye imaginal disc. Oncogene 18: 6767-6775

31. Patel $S$ and Latterich M (1998) The AAA team: related ATPases with diverse functions. Trends Cell Biol. 8: 65-71

32. Dai R-M, Chen E, Longo DL, Gorbea CM and Li C-CH (1998) Involvement of Valosin-containing Protein, an ATPase Co-purified with $I_{\kappa} \mathrm{B} \alpha$ and $26 \mathrm{~S}$ Proteasome, in Ubiquitin-Proteasome-mediated Degradation of $I_{\kappa} \mathrm{B} \alpha$. J. Biol. Chem. 273: 3562-3573

33. Leon A and Mckearin D (1999) Identification of TER94, an AAA ATPase protein, as a Bam-independent component of the Drosophila fusome. Mol. Biol. Cell 10: $3825-3834$

34. Madeo F, Frohlich E and Frohlich KU (1997) A yeast mutant showing diagnostic markers of early and late apoptosis. J. Cell Biol. 139: 729-734

35. Shirogane T, Fukada T, Muller JM, Shima DT, Hibi M and Hirano T (1999) Synergistic roles for Pim-1 and c-Myc in STAT3-mediated cell cycle progression and antiapoptosis. Immunity 11: 709-719

36. Hirabayashi M, Inoue K, Tanaka K, Nakadate K, Ohsawa Y, Kamei Y, Popiel AH, Sinohara A, Iwamatsu A, Kimura Y, Uchiyama Y, Horil S and Kakizuka A (2001) $\mathrm{VCP} / \mathrm{p} 97$ in abnormal protein aggregates, cytoplasmic vacuoles, and cell death, phenotypes relevant to neurodegeneration. Cell Death Differ. 8: 977-984

37. Latterich M, Frohlich KU and Schekman R (1995) Membrane fusion and the cell cycle: Cdc48p participates in the fusion of ER membranes. Cell 82: 885-893

38. Madeo F, Schlauer J, Zischka H, Mecke D and Frohlich KU (1998) Tyrosine phosphorylation regulates cell cycle-dependent nuclear localization of Cdc48p. Mol. Biol. Cell 9: $131-141$

39. Rabouille C, Levine TP, Peters JM and Warren G (1995) An NSF-like ATPase, p97, and NSF mediate cisternal regrowth from mitotic Golgi fragments. Cell 82 : 905-914

40. Ikeda H, Yamaguchi M, Sugai S, Aze Y, Narumiya S and Kakizuka A (1996) Expanded polyglutamine in the Machado-Joseph disease protein induces cell death in vitro and in vivo. Nat. Genet. 13: 196-202

41. Yasuda S, Inoue K, Hirabayashi M, Higashiyama H, Yamamoto $\mathrm{Y}$, Fuyuhiro $\mathrm{H}$, Komure O, Tanaka F, Sobue G, Tsuchiya K, Hamada K, Sasaki H, Takeda K, Ichijo $\mathrm{H}$ and Kakizuka A (1999) Triggering of neuronal cell death by accumulation of activated SEK1 on nuclear polyglutamine aggregations in PML bodies. Genes Cells 4: 743-756

42. Hay BA, Wolf T and Rubin GM (1994) Expression of baculovirus P35 prevents cell death in Drosophila. Development 120: 2121-2129 